\title{
Transcatheter Intervention for Paravalvular Leak in Mitroflow Bioprosthetic Pulmonary Valve
}

\author{
Vishal R. Kaley, MBBS, MD1, E. Oliver Aregullin, MD, FAAP ${ }^{1,2}$, Bennett P. Samuel, MHA, BSN, RN ${ }^{1}$, \\ Joseph Vettukattil, MBBS, MD, DNB, CCST, FRCPCH ${ }^{1,2^{*}}$ \\ ${ }^{1}$ Congenital Heart Center, Spectrum Health Helen DeVos Children's Hospital, Grand Rapids, Michigan, USA \\ 2 Pediatrics and Human Development, College of Human Medicine, Michigan State University, Grand Rapids, Michigan, USA
}

\begin{abstract}
Paravalvular leak (PVL) is a complication caused by development of gaps due to dehiscence between the annulus and implanted valve. Clinically significant PVL in bioprosthetic pulmonary valves are extremely rare. Currently, surgical intervention is the first line of treatment. However, surgery is associated with greater risk for morbidity and mortality when compared to primary repair or valve replacement. We present a 22-yearold male who underwent successful transcatheter intervention for pulmonary PVL with hemodynamic and symptomatic improvement.

Copyright @ 2019 Science International Corp.
\end{abstract}

\section{Key Words}

Paravalvular leak - Percutaneous - Pulmonary valve replacement • Regurgitation • Tetralogy of Fallot.

\section{Introduction}

Paravalvular leak (PVL) is a known complication due to suture dehiscence between the sewing ring and native tissue causing regurgitation around the replaced valve [1]. Large leaks may result in complications such as hemolysis, arrhythmias, and congestive heart failure [2]. The incidence of PVL following aortic and mitral valve replacement is $1-5 \%$, and $2-12 \%$ respectively [1]. Clinically significant PVL following bioprosthetic pulmonary valve replacement is extremely rare.

Fax +1 2037853346

E-Mail: jshd@scienceinternational.org

http://structuralheartdisease.org/

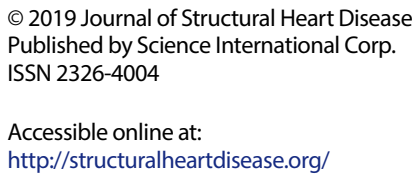

(c) 2019 Journal of Structural Heart Disease Published by Science International Corp. ISSN 2326-4004

Accessible online at: http://structuralheartdisease.org/

The standard treatment for pulmonary PVL is surgery with valve replacement and repair due to the variability in implant location in the trabeculated and often dilated right ventricular (RV) outflow tract [3]. However, surgery is associated with greater risk for morbidity and mortality when compared to primary repair or valve replacement [4]. Transcatheter intervention for aortic and mitral valve PVL is effective with low rates of procedural complications [5], and may also be useful in the setting of pulmonary PVL.

\section{Case Presentation}

A 22-year-old male with tetralogy of Fallot and bilateral peripheral pulmonary artery (PA) stenosis presented with multiple episodes of syncope, chronic dyspnea on exertion and worsening lower extremity edema. He had a transannular patch repair early in life. Due to severe pulmonary regurgitation, his pulmonary valve was replaced with a $27 \mathrm{~mm}$ Mosaic tissue valve (Medtronic, Minneapolis, MN, USA) at 8-years of age. He was noted to have free pulmonary regurgitation, and depressed systolic function at 15-years of age. Subsequently, he underwent pulmonary valve replacement with a $25 \mathrm{~mm}$ Mitroflow bioprosthetic valve (Sorin, Saluggia, Italy) and intraoperative stenting of the branch PAs. His post-operative period was complicated requiring extracorporeal membrane

* Corresponding Author:

Joseph Vettukattil, MBBS, MD, DNB, CCST, FRCPCH Congenital Heart Center

Spectrum Health Helen DeVos Children's Hospital

100 Michigan NE (MC248), Grand Rapids, MI , USA

Tel. +1 616267 0988; Fax: +1 616267 1408;

E-Mail: joseph.vettukattil@helendevoschildrens.org 


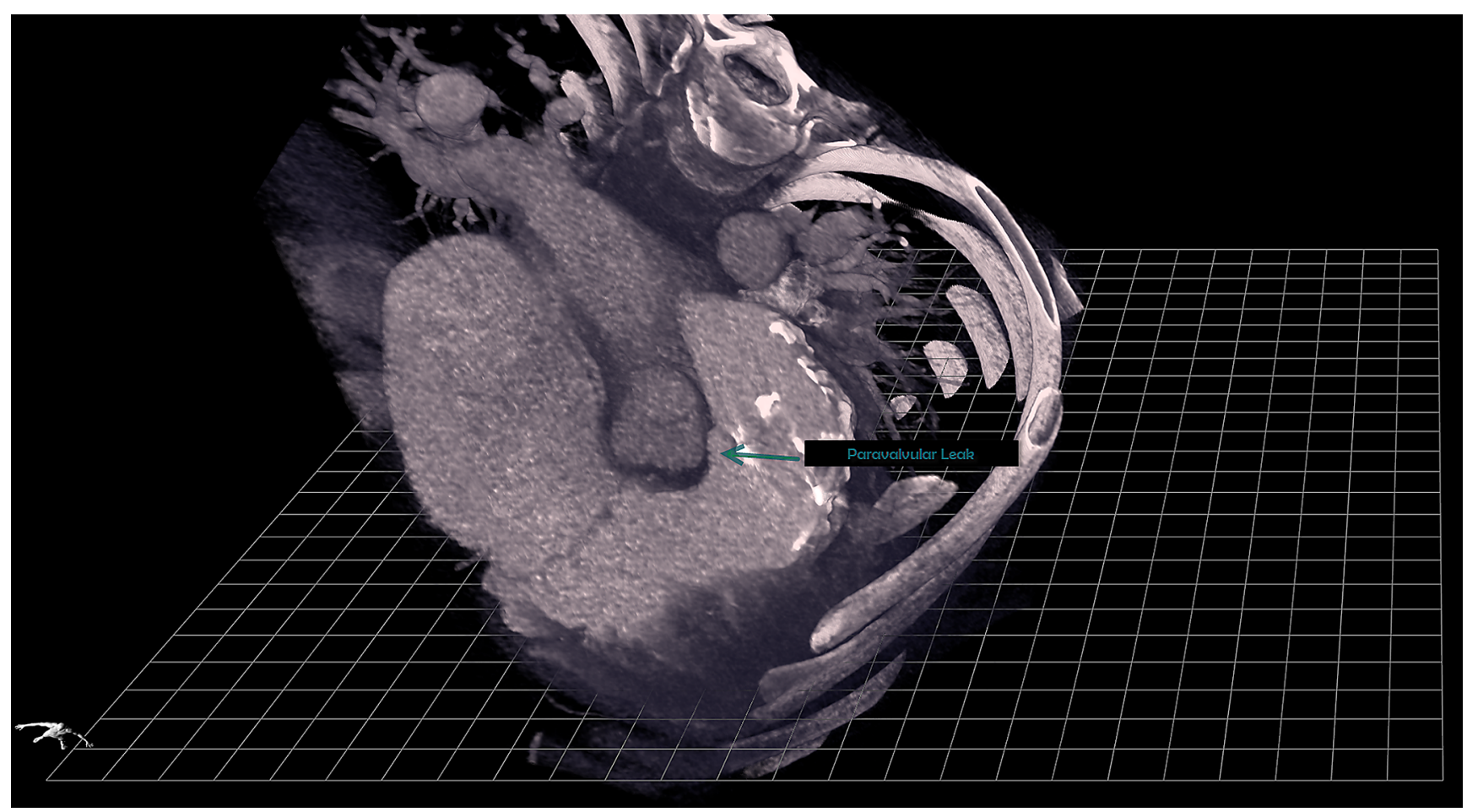

Figure 1. Computed tomography viewed on True3D Viewer (Echopixel, Inc., Mountain View, CA) showing paravalvular tunnel in the posteromedial side of the pulmonary valve.

oxygenation support and prolonged tracheostomy leading to severe post-traumatic stress syndrome.

On recent evaluation, computed tomography scan showed grossly calcified valve leaflets with a PVL tunnel measuring $8 \times 6 \mathrm{~mm}$ and approximately $9 \mathrm{~mm}$ in length in the posteromedial side of the pulmonary valve adjacent to the right aortic sinus. Moderate RV hypertrophy and mildly dilated RV volume $(132 \mathrm{~mL} /$ $\mathrm{m}^{2}$ ) was noted (Figure 1). He developed symptomatic ventricular tachycardia. Considering his complex history and associated risks with redo sternotomy, a multi-disciplinary team recommended transcatheter PVL closure.

A complete right and left heart catheterization was performed. Moderate stenosis was noted across the Mitroflow valve at the pulmonary position with gradient of $25-30 \mathrm{mmHg}$. The RV pressure was supra-systemic measured at $86 / 11 \mathrm{mmHg}$ as compared to the right femoral artery pressure of $74 / 47 \mathrm{mmHg}$. Elevated PA pressure of $56 / 6(28) \mathrm{mmHg}$ with mean pulmonary capillary wedge pressure of $12 \mathrm{mmHg}$ was noted. The pulmonary vascular resistance was 5.2 Wu/m² with Qp:Qs of 1:1. PA angiography demonstrated moderate pulmonary insufficiency and PVL. The pulmonary PVL site was localized by balloon occlusion of the valve and simultaneous contrast injection into the PA (Figure 2A). Subsequently, a 0.035 inch glidewire was positioned across the PVL. The defect was sized using an Armada balloon (Abbott, Abbott Park, IL, USA; 8x4 mm; Figure 2B). After careful hemodynamic and angiographic evaluation, a $12 \mathrm{~mm}$ Amplatzer vascular plug II (AVP II; Abbott, Abbott Park, IL, USA) was deployed in the tunnel-like leak without any complications or significant residual leak (Figure 2C). The diastolic PA pressure improved from 6 to $20 \mathrm{mmHg}$ after PVL closure. The patient recovered well and was discharged to home the following day. The 6-week post-procedure transthoracic echocardiogram showed peak velocity of $3.2 \mathrm{~m} / \mathrm{sec}$ with peak gradient of $42 \mathrm{mmHg}$ and mean gradient of 24 $\mathrm{mmHg}$ through the pulmonary valve with moderate pulmonary regurgitation. 


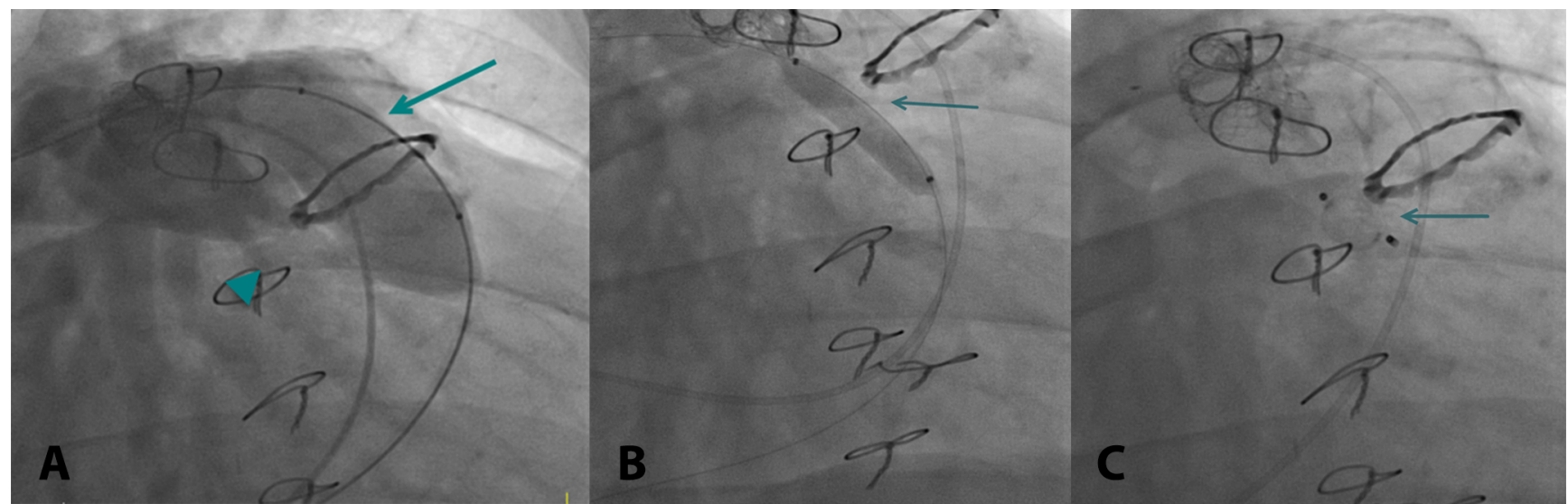

Figure 2. Panel A. Arteriogram showing the PVL (arrow head) after balloon occlusion (arrow) of the pulmonary bioprosthetic valve; Panel B. PVL size confirmed using Armada balloon $(8 \times 4 \mathrm{~mm})$ passed across the leak; Panel C. 12 $\mathrm{mm}$ AVP II successfully deployed in the tunnel-like leak.

\section{Discussion}

The standard treatment for pulmonary PVL is surgical intervention with valve replacement and repair [3]. However, surgery is associated with a higher risk for morbidity and mortality than primary repair or valve replacement. In a study of 122 patients with PVL (mitral $=67.2 \%$, and aortic $=32.8 \%$ ), the 12-year survival following surgery was about $30 \%-40 \%$ with high rates of recurrence [4]. Transcatheter intervention in the setting of bioprosthetic mitral and aortic valve PVL has been shown to have better long-term outcomes than surgery [5]. The AVP II is the most common device used for mitral and aortic valve PVL due to the shape of its discs resulting in good epithelization and providing an optimal seal. Other off-label devices that may be used for PVL closure by physician discretion and expertise are ventricular septal defect, atrial septal defect, and patent ductus arteriosus occluders, AVP I, and AVP III [1]. However, no dedicated catheters or devices are currently approved for PVL closure by the U.S. Food and Drug Administration.

Although transcatheter closure of pulmonary PVL are previously reported using the AVP II and ventric- ular septal defect occluders [2, 3], it is associated with risks including impingement of valve leaflets, device embolization, hemolysis, pericardial effusion, and arrhythmias [1]. Successful transcatheter pulmonary PVL closure is dependent on appropriate case selection and operator expertise. The alternative to device occlusion is to use a covered stent in anatomically appropriate conduit with placement of transcatheter valve in the stent.

Pulmonary PVL following bioprosthetic valve implantation is rare, but can be a hemodynamically significant complication. Transcatheter intervention is a feasible treatment option with lower complication rates than surgery and must be considered in the setting of pulmonary PVL.

\section{Conflict of Interest}

The authors have no conflict of interest relevant to this publication.

Comment on this Article or Ask a Question 


\section{References}

1. Reed GW, Tuzcu EM, Kapadia SR, Krishnaswamy A. Catheter-based closure of paravalvular leak. Expert Rev Cardiovasc Ther. 2014;12:681-692. DOI: 10.1586/14779072.2014.915193

2. Seery TJ, Slack MC. Percutaneous closure of a prosthetic pulmonary paravalvular leak. Congenit Heart Dis. 2014;9:E19-22. DOI: 10.1111/chd.12058

3. Chikkabyrappa S, Mosca RS, McElhinney DB. Retrograde transcatheter device closure of a complex paravalvular leak after bioprosthetic pulmonary valve replacement in a pediatric patient. Catheter Car- diovasc Interv. 2016;87:1294-1297. DOI: $10.1002 / \mathrm{ccd} .26316$

4. Taramasso M, Maisano F, Denti P. Surgical treatment of paravalvular leak: Longterm results in a single center experience (up to14 years). J Thorac Cardiovasc Surg. 2015;149:1270-1275. DOI: 10.1016/j. jtcvs.2014.12.041

5. Angulo-Llanos R, Sarnago-Cebada F, Rivera $A R$, et al. Two-year follow up after surgical versus percutaneous paravalvular leak closure: A non-randomized analysis. Catheter Cardiovasc Interv. 2016;88:626-634. DOI: $10.1002 / \mathrm{ccd} .26459$
Cite this article as: Kaley VR, Aregullin EO, Samuel BP, Vettukattil J. Transcatheter Intervention for Paravalvular Leak in Mitroflow Bioprosthetic Pulmonary Valve. Structural Heart Disease. 2019;5(1):21-24. DOI: https://doi. org/10.12945/j.jshd.2018.003.18 